\title{
Numerical investigation of noise induced changes to the solution behaviour of the discrete FitzHugh-Nagumo equation
}

\author{
Neville J. Ford ${ }^{\mathrm{a}}$, Pedro M. Lima ${ }^{\mathrm{b}, *}$, Patricia M. Lumb ${ }^{\mathrm{a}}$ \\ ${ }^{a}$ Dept. of Mathematics, University of Chester, CH1 4BJ, Chester, UK \\ ${ }^{b}$ CEMAT, Instituto Superior Técnico, University of Lisbon, 1049-001 Lisboa, Portugal
}

\begin{abstract}
In this work we introduce and analyse a stochastic functional equation, which contains both delayed and advanced arguments. This equation results from adding a stochastic term to the discrete FitzHugh-Nagumo equation which arises in mathematical models of nerve conduction. A numerical method is introduced to compute approximate solutions and some numerical experiments are carried out to investigate their dynamical behaviour and compare them with the solutions of the corresponding deterministic equation.
\end{abstract}

Keywords: Discrete FitzHugh-Nagumo equation, stochastic mixed-type functional differential equation, Euler-Maruyama method 2000 MSC: 34K50, 65C30, 65C20

\section{Introduction}

The main purpose of this paper is to analyse a stochastic mixed-type functional differential equation, which may be written in the form:

$$
d y(t)=[A(y(t+\tau)-2 y(t)+y(t-\tau))+B y(t))(y(t)-1)(\alpha-y(t))] d t+\gamma y(t)(y(t)-1) d W(t),
$$

where $\alpha, A, B, \tau$ are known real parameters. In the next section we will discuss in detail the boundary conditions we consider in the paper. As far as we are aware, the subject of stochastic mixed-type functional differential equations (MTFDE) is new in the literature and we begin by explaining our motivation to introduce this type of equation. In some recent works [10] [18] we were concerned about a deterministic MTFDE:

$$
y^{\prime}(t)=A[y(t+\tau)-2 y(t)+y(t-\tau)]+B y(t)(y(t)-1)(\alpha-y(t)),
$$

sometimes known as the discrete FitzHugh-Nagumo equation, arising in nerve conduction theory. The history of the analysis of this equation began in 1952, when A. Hodgkin and A. Huxley [13] introduced a mathematical model that describes the excitation and flow of electrical current through the surface of a giant nerve fibre from a squid. This investigation was continued in the works of FitzHugh [7], [8] and Nagumo $[25]$.

During its evolution the FitzHugh-Nagumo equation has taken different forms, and after various simplifications it appeared in the work [6] in the form (2), where $y(t)$ represents the potential at a Ranvier node of

\footnotetext{
* Corresponding author

Email addresses: njford@chester.ac.uk (Neville J. Ford), plima@math.ist.utl.pt (Pedro M. Lima), plumb@chester.ac.uk (Patricia M. Lumb)
} 
the axon at the moment $t$ (in this case, the potential at the neighbouring nodes is denoted by $y(t-\tau)$ and $y(t+\tau)$; the constant $\tau$ is the time that a signal takes to be transmitted from a node to the neighbouring one (in other words, $\tau$ is inversely proportional to the propagation speed of the signal). The constants $A, B$ reflect the resistance and the conductance in the nerve axon, while $\alpha$ is the threshold potential.

From a mathematical point of view, an important feature of equation (2) is that it contains both negative and positive deviations of the argument (delayed and advanced terms); this is the reason it is called a mixed type functional differential equation (or an advance-delay-differential equation). Important contributions to the analysis of this type of equation have appeared in the literature in the last two decades of the past century. The ill-posedness of mixed-type functional differential equations was discussed by Rustichini in [30], where he considered linear autonomous equations. He extended his results to nonlinear equations [31]. Mallet-Paret applied Fredholm theory to obtain new results for this class of equation [21] and introduced the idea of factorisation of their solutions [22]. More recently, Hupkes and Verduyn Lunel studied the behaviour of solutions to nonlinear autonomous mixed-type functional differential equations in the neighbourhood of an equilibrium solution [14]. Based on existing insights into the qualitative behaviour of MTFDEs, the authors of [9] developed a new approach to the analysis of these equations in the autonomous case. More precisely, they analysed MTFDEs as boundary value problems, that is, for a linear MTFDE they considered the problem of finding a differentiable solution on a certain real interval $[-1, k], k \in \mathbb{N}$, given its values on the intervals $[-1,0]$ and $(k-1, k]$. This approach was developed further in [32], where new numerical methods were proposed for the solution of such boundary value problems. In [17] and [11], these methods were extended to the non-autonomous case and new results were obtained about their numerical analysis. Once efficient computational methods had been created for the numerical treatment of linear MTFDEs, the next step was to extend these methods to the case of nonlinear ones, which includes equations of the form (2). This was done by Abell et al. [1] and then by the authors of the present paper in [18] and [10].

Up to now we have been concerned only with deterministic equations such as (2). However the study of stochastic problems such as (1) is of great importance for the modelling of excitable neural systems. Noise-induced effects on nonlinear systems have attracted great research interest in the last 20 years. In particular scientists have been interested in stochastic resonance, which occurs when a periodic input signal in a nonlinear system can be amplified under the effect of noise. In some cases, noise can play a constructive role in excitable neural systems, even when there is no deterministic input signal; this phenomenon is named coherence resonance. Both stochastic and coherence resonance can be enhanced when a resonator is coupled into a chain of identical ones. In [19] the authors show how noise, coupling and bi-stable dynamics cooperate to organise spacial order, temporal periodicity and peak signal-to-noise ratio. The effect of coherence resonance in a heterogeneous array of coupled FitzHugh-Nagumo neurons in demonstrated in [33]. In both cited works only one-dimensional arrays of excitable neurons are considered, such that each neuron can interact only with two neighbours. This case is modelled by equations similar to (1). The case of two-dimensional arrays has been also considered (see for example [27]). In that paper the author shows that there is an optimal noise intensity, for which the inherent spatial periodicity of the system is resonantly pronounced. In the two-dimensional case the focus is often shifted towards networks with variable random connectivity. In these systems, known as small-world networks, there is a certain fraction of socalled long-range couplings (or shortcut links) that connect distant units of the system, while all other units are coupled in a diffusive-like manner. In [16] the authors investigate the effect of small-world connectivity on the phenomenon of coherence resonance of Hodgkin-Huxley neurons. In [28] it is shown that that the temporal order, characterised by the auto-correlation of a firing-rate function, can be enhanced by smallworld connectivity. On the other hand, the introduction of long-range couplings induces disorder in otherwise ordered noise-induced patterns that can be observed in the case of exclusively diffusive connectivity. This latter effect, known as spatial decoherence, is studied in detail in [29].

In our work we aim to compare the dynamical behaviour of the solutions to equation (1) with those of 
(2). A similar comparison has been carried out in [26], in the case of a stochastic delay-differential equation.

This is a very challenging aim, since (as far as we know) there have been no previous attempts at investigating stochastic mixed-type functional differential equations. Therefore, we must rely on insights available from the related field of stochastic delay differential equations.

In the last ten years many works have been devoted to the analysis of different numerical algorithms applied to stochastic delay-differential equations, including the Euler-Maruyama [4], [20],[23], [24], explicit one-step [2], Maruyama multistep [5] and Milstein methods [15].

The outline of this paper is as follows. In Sec. 2 we recall the most important features of the deterministic equation (2) and introduce numerical methods for its approximation. In Sec. 3 we introduce the stochastic equation (1) and describe a numerical method for its approximation, based on the algorithm for the deterministic equation. In Sec. 4 we present and discuss numerical results. We finish with some conclusions in Sec. 5 .

\section{Analysis and approximation of the deterministic equation}

\subsection{Building the forward and backward solutions}

We consider the following equation:

$$
y^{\prime}(t)=A(y(t+\tau)-2 y(t)+y(t-\tau))+B f(y(t))
$$

where $A, B, \tau$ are given constants,

$$
f(x)=x(x-1)(\alpha-x),
$$

with a certain $\alpha(0<\alpha<1)$. In [10] and [18] boundary value problems (BVP) were considered for equations of this type, where the solution is required to satisfy the following boundary conditions:

$$
\lim _{t \rightarrow \infty} y(t)=1, \quad \lim _{t \rightarrow-\infty} y(t)=0, \quad y(0)=1 / 2
$$

It is known (see for example [6]) that if $0<\alpha<0.5$, a BVP of the form (3)-(5) has a unique solution in the set of monotone functions for a certain value of the argument deviation $\tau$.

Numerical methods have been developed for the solution of such a BVP, including finite-difference methods [6], [10], and collocation methods [18].

In the present paper we are concerned with initial value problems for equation (3),(4) with different sets of initial conditions.

In the first case (forward approximation) we will suppose that an initial approximation is given on $[-3 \tau,-2 \tau]$. Following the approach in [6], we assume that on this interval the approximation has the form

$$
v(t)=\epsilon_{1} e^{\lambda_{+}(t+3 \tau)},
$$

where $\epsilon_{1}$ is an adjustable parameter and $\lambda_{+}$is a positive root of the characteristic equation of (3), obtained when this equation is linearised, as $t \rightarrow-\infty$. This assumption is based on the asymptotic behaviour of the solutions which satisfy the second part of condition (5) (this was analysed in detail in [6] and [18]). Note that the choice of $-3 \tau$ as the lower limit of the approximation domain is, to a certain degree, arbitrary. We have chosen this value based on our previous experience (see [10] and [18]), where we have obtained evidence that this length of interval, in most cases of interest, is large enough to guarantee that the expression (6) approximates the exact solution with reasonable accuracy. The application of the method on a larger interval of approximation would increase the effect of numerical instability (which can be very strong, as we shall see in the sequel). 
Next we will describe a corresponding method that we have applied to approximate the solution on the positive semiaxis. In this case, we start with the asymptotic approximation of the solution, which is known as $t \rightarrow \infty$, and we build the solution backwards. Analogously to (6), on $[2 \tau, 3 \tau]$ we represent the solution as

$$
w(t)=1-\epsilon_{2} e^{\lambda_{-}(t-3 \tau)},
$$

where $\tau$ has the same value as in $(6) ; \lambda_{-}$is a negative root of the characteristic equation, as $t \rightarrow \infty$, of the linearised version of (3). We assume that (7) gives a good approximation of the exact solution of (3), based on the asymptotic analysis of the solutions which satisfy the condition $\lim _{t \rightarrow \infty} y(t)=1$.

We know from previous work [6] that for each $\alpha(0<\alpha<0.5)$, there exists a unique $\tau>0$ and a unique monotone solution $y$ which satisfies equation (3) and conditions (5).

Therefore a solution of an initial value problem, resulting from forward or backward approximation (as described above), does not necessarily correspond to a solution of the BVP (3),(5). This happens only if the correct value of $\tau$ is known. Since in the present work we do not attempt to determine the value of $\tau$, in order to guarantee that the approximate solutions we obtain have some physical meaning we rely in the values of $\tau$ obtained in previous works (see [6], [10]). Note also that once the value of $\tau$ is known, the values of $\epsilon_{1}$ and $\epsilon_{2}$ in (6) and (7) can be determined, as well as the roots $\lambda_{+}$and $\lambda_{-}$.

\subsection{Numerical Approximation}

We now describe a discretisation of equation (3) and the resulting numerical algorithm. We remark that our final goal is to analyse a stochastic differential equation, arising as a perturbation of equation (3), and therefore our approach is based on the approximation of initial value problems (rather than a boundary value problem, as considered in [18] and [10], for example). This will enable us to introduce classical numerical methods for stochastic differential equations in the next section.

We start by introducing a uniform mesh on $[-3 \tau, 3 \tau]$ with stepsize $h=\tau / N$, for a given $N$. Then we have $t_{i}=-3 \tau+i h, i=0, \ldots, 6 N$. Using a forward difference to approximate $y^{\prime}(t)$ in $(3)$, and denoting $y_{i}$ as the approximation of $y\left(t_{i}\right), i=0, \ldots, 6 N$, we obtain the following difference equation:

$$
\frac{y_{i+1}-y_{i}}{h}=A\left(y_{i+N}-2 y_{i}+y_{i-N}\right)+B f\left(y_{i}(t)\right) .
$$

We could try to rearrange equation (8) to determine $y_{i+N}$, assuming that $y_{i+1}, y_{i}, y_{i-1}$, and $y_{i-N}$ are known (this is similar to the approach in [18]). However, in this case, this results in a highly unstable multistep method. Therefore, we have decided to look at equation (8) as the discretisation of a delay differential equation and to treat the advanced term by means of an iterative procedure.

Suppose that an initial approximation $y^{(0)}$ to the solution of $(3)$ is known on the interval $[-3 \tau, 3 \tau]$. In our computations, these initial approximations were taken from the numerical results in [10]. If we replace in (8) the term $y_{i+N}$ by $y_{i+N}^{(0)}$, and any other entry $y_{j}$ by $y_{j}^{(1)}$ we obtain:

$$
\frac{y_{i+1}^{(1)}-y_{i}^{(1)}}{h}=A\left(y_{i+N}^{(0)}-2 y_{i}^{(1)}+y_{i-N}^{(1)}\right)+B f\left(y_{i}^{(1)}\right), \quad i=N+1, \ldots, 4 N .
$$

This equation gives a recursive formula to compute all the entries $y_{i}^{(1)}, i=N+1, \ldots, 4 N$, from left to right. Equation (9) is similar to the classical Euler Method, used to approximate the solutions of delay differential equations. Solving this equation one obtains a new approximation $y^{(1)}$ to the solution of equation (3). For $i=1, . ., N$ and $i=4 N+1, \ldots, 5 N$ we set $y_{i}^{(1)}=y_{i}^{(0)}$. In this way, a new approximate solution on the interval $[-3 \tau, \tau]$ is obtained. Moreover, this process can be iterated:

$$
\frac{y_{i+1}^{(k+1)}-y_{i}^{(k+1)}}{h}=A\left(y_{i+N}^{(k)}-2 y_{i}^{(k+1)}+y_{i-N}^{(k+1)}\right)+B f\left(y_{i}^{(k+1)}\right), \quad i=N+1, N+2, \ldots, 4 N ;
$$


New iterates $y^{(k+1)}$ are computed by this formula until the stopping criterion is satisfied:

$$
\left\|y^{(k+1)}-y^{(k)}\right\|<\epsilon,
$$

for a given $\epsilon$.

Note that a similar approach can be applied to build a 'backward' approximation. In this case, we assume that the initial approximation $y^{(0)}$ is known on the interval $[-\tau, 3 \tau]$. Instead of the iterative formula $(10)$, we use the formula

$$
\begin{gathered}
\frac{y_{i-1}^{(k+1)}-y_{i}^{(k+1)}}{h}=-A\left(y_{i+N}^{(k+1)}-2 y_{i}^{(k+1)}+y_{i-N}^{(k)}\right)-B f\left(y_{i}^{(k+1)}\right), \\
i=4 N, 4 N-1, \ldots, N+1, \quad k=1,2, \ldots
\end{gathered}
$$

In this case, the approximate solution is computed from right to left.

\subsection{On the convergence of the iterative process}

In the previous subsection we have introduced an iterative procedure to solve numerically the nonlinear system of equations (8). The convergence analysis of this iterative process is a complex theoretical problem. If, for example, the values of $y_{i}$ are known for $i=0, \ldots, N$ and $i=3 N+1, \ldots, 4 N$, the formulae (8) can be seen as a system of $2 N$ nonlinear equations, with respect to the unknowns $y_{i}, i=N+1, \ldots, 3 N$. If $B=0$, then this system is linear and the proposed iterative procedure is equivalent to the Gauss-Seidel method, which converges under well-known conditions. In the nonlinear case, in order to analyse the convergence of the proposed iterative procedure one has to write equations (10) in the form

$$
y^{(k+1)}=G\left(y^{(k)}\right),
$$

where $y^{(k)}$ denotes the $k$ - th iterate of the unknown vector and $G$ is a nonlinear function of $2 N$ variables, for which the exact solution of the nonlinear system is a fixed point. Note that $G$ has a linear part, governed by the coefficient $A$, and a nonlinear one, governed by $B$ (see (3)).

Fixed point theory can be used to establish conditions under which the iterative process (12) converges. Such analysis is beyond the scope of the present paper. We note that the choice of an initial approximation $y^{(0)}$, sufficiently close to the solution that we seek, is an important condition for convergence. We also remark that the forward formula (10) and the backward formula (11) give rise to independent iterative processes (though the underlying system of equations is the same). Therefore, it may happen that for a particular case one of the processes converges but the other does not.

In the numerical simulations that we will describe in Sec.4, we have taken an experimental approach. As mentioned before, we have chosen as initial conditions solutions obtained by means of other methods. In each case we have implemented the forward and the backward iterative processes. The process has been considered as convergent when the stopping criterion was attained within 30 iterations. In all the cases presented here, this has happened for at least one of the iterative processes (forward or backward). The number of iterations is between 5 and 7 , in the case of $\epsilon=10^{-5}$. The same is true in the case of the stocastic equation treated in the next section.

\section{The stochastic equation and its numerical approximation}

When modelling the propagation of nerve impulses it is natural to assume that the propagated signal is affected by random effects. Such effects may be taken into consideration by introducing a stochastic term 
in equation (3). More precisely, knowing that the threshold potential $\alpha$ in (4) is a parameter that can be determined only experimentally, we can replace the function $f$, defined by (4), by a certain $\tilde{f}$, such that

$$
\tilde{f}(x)-f(x)=(\tilde{\alpha}-\alpha) x(x-1)
$$

then, assuming that $\tilde{\alpha}-\alpha$ is a random variable, we write the stochastic term in the form

$$
\Gamma(t)=\gamma y(t)(y(t)-1) W(t),
$$

where $\gamma$ is a positive constant and $W(t)$ is a stochastic process with normal distribution, zero expectation and standard deviation 1 , that is $W(t) \sim N(0,1)$. Finally, if in $(3)$ we replace $B f(y)$ by $B f(y)+\Gamma(t)$, we obtain the following stochastic equation

$$
d y(t)=(A(y(t+\tau)-2 y(t)+y(t-\tau))+B f(y(t))) d t+\gamma y(t)(y(t)-1) d W(t) .
$$

In the remainder of this work we analyse (15) and related equations and compare their numerical solutions with those of the corresponding deterministic equations. To simplify, we assume that

$$
W(t)=0, \quad \text { if } \quad t<-2 \tau \quad \text { or } \quad t>2 \tau,
$$

that is, the solution is not affected by noise outside of $[-2 \tau, 2 \tau]$.

In order to obtain numerical approximations of (15) we use the Euler-Maruyama method (see, for example, [12]). In this case, instead of (9), when we compute the numerical solution forwards we use the equation

$$
\begin{gathered}
y_{i+1}^{(k+1)}=y_{i}^{(k+1)}+h\left(A\left(y_{i+N}^{(k)}-2 y_{i}^{(k+1)}+y_{i-N}^{(k+1)}\right)+B f\left(y_{i}^{(k+1)}\right)\right)+\gamma \sqrt{h} y_{i}^{(k+1)}\left(y_{i}^{(k+1)}-1\right) w_{i}, \\
i=N+1, N+2, \ldots
\end{gathered}
$$

where $w_{i}$ represents a random variable with standard normal distribution $N(0,1)$. Equation (17) can be used to compute each trajectory of the numerical solution on the interval $[-3 \tau, 0]$, provided that an initial approximation $y^{0}$ is given on $[-3 \tau, \tau]$. As an initial approximation we take an approximation to the monotone solution of the deterministic equation (3), obtained by a different numerical method. Analogously, assuming that an initial approximation $y^{(0)}$ is given on $[-\tau, 3 \tau]$, the numerical solution on $[0,2 \tau]$ could be computed backwards, using the formula

$$
\begin{gathered}
y_{i-1}^{(k+1)}=y_{i}^{(k)}-h\left(A\left(y_{i+N}^{(k+1)}-2 y_{i}^{(k+1)}+y_{i-N}^{(k)}\right)-B f\left(y_{i}^{(k+1)}\right)\right)+\gamma \sqrt{h} y_{i}^{(k+1)}\left(y_{i}^{(k+1)}-1\right) w_{i}, \\
i=3 N, 3 N-1, \ldots, 0 .
\end{gathered}
$$

\section{Numerical results}

\subsection{Perturbation of a constant solution}

It is evident that $y(t) \equiv \alpha$ is a solution of equation (3), which is an unstable equilibrium solution of this equation. The equation also has the stable equilibrium solutions $y(t) \equiv 0$ and $y(t) \equiv 1$. In this subsection we shall answer the question: how does the solution $y(t) \equiv \alpha$ change if equation (3) is perturbed?

With this in mind, we apply the method (10) to the solution of the deterministic equation

$$
y^{\prime}(t)=A(y(t+\tau)-2 y(t)+y(t-\tau))+B f(y(t))+\gamma y(t)(y(t)-1)
$$


which is a perturbation of (3) (the nonlinear term $B f(y(t)$ is replaced by $B f(y(t))+\gamma y(t)(y(t)-1)$ ); in this case, we assume that the solution satisfies the condition $y(t) \equiv \alpha$, if $t \in[-3 \tau,-2 \tau]$.

Next we consider the stochastic equation (15) and compute a solution which satisfies the conditions

$$
y(t) \equiv \alpha, \quad \text { if } t \in[-3 \tau,-2 \tau], \quad y(t) \equiv 0.5, \quad \text { if } t \in[2 \tau, 3 \tau] .
$$

The reason for the second boundary condition will be explained below, during the discussion of the numerical experiments. The value of $\tau$ in all the examples of this subsection is 1 . We take the other parameters of equation (15) as $A=1$ and $B=15$, except where other values are specified. We apply the numerical method (17) using as initial approximation $y^{(0)}(t) \equiv \alpha$. In this way we obtain a numerical solution on the interval $(-2 \tau, 2 \tau)$.

Now the constant solution $y(t) \equiv \alpha$ is an unstable stationary point of equation (3), so we know that even a small perturbation of the equation may produce a completely different solution, either in the deterministic or in the stochastic case. This is one of the issues we want to investigate through our numerical experiments.

With the purpose of answering this question, we applied the method (17) to solve equation (15), with boundary conditions (20), for different values of $\alpha$ and $\gamma$. We also applied the method (10) to solve the deterministic equation (19), with $y(t) \equiv \alpha$, if $t \in[-3 \tau,-2 \tau]$. When $\gamma>0$ (the threshold potential is increased), it is to be expected that the constant solution of (19) will be transformed into a solution which is less than $\alpha$ on $[-2 \tau, 2 \tau]$; therefore we set the second boundary condition $y(t) \equiv 0$, if $t \in[2 \tau, 3 \tau]$. When $\gamma<0$ (the threshold potential) is decreased, it is to be expected that the constant solution of (19) will be transformed into a solution,which is greater than $\alpha$ on $[-2 \tau, 2 \tau]$; therefore we set the second boundary condition $y(t) \equiv 1$, if $t \in[2 \tau, 3 \tau]$. Note that 0 and 1 are the stable points of the equation. On the other hand, if we consider the stochastic equation (15) with the same boundary condition, and a certain value of $\gamma$, it is to be expected that the trajectories of its solutions are located between the solutions of the deterministic equations corresponding to $\gamma$ and $-\gamma$. This is illustrated by Fig. 1. In the stochastic case, besides the expected value of the solution $E(y(t))$, we have considered the functions

$$
y_{\min }(t)=\min _{i=1, ., n s} y_{(i)}(t),
$$

and

$$
y_{\text {max }}(t)=\max _{i=1, . ., n s} y_{(i)}(t),
$$

where $y_{(i)}$ denotes the $i_{t h}$ trajectory of a solution of the stochastic equation and $n s$ is the total number of trajectories. Note that in all the graphs in the present work the horizontal axis corresponds to the independent variable $t$, while the vertical one corresponds to $y, y_{\min }, y_{\max }$ or $E(y)$. Comparing Fig.1,2 and 3 , we conclude that $y_{\min }(t)$ has a similar behaviour to the deterministic solution, corresponding to $\gamma=0.001$, while $y_{\max }(t)$ has a similar behaviour to the deterministic solution, corresponding to $\gamma=-0.001$.

Moreover, these figures show that the constant solution is indeed very sensitive to small perturbations of the equation. Actually, even with $\gamma=0.001$, for $\alpha=0.2$, in the deterministic case, we get modified solutions that tend to 0 (when $\gamma>0$ ) or to 1 (when $\gamma<0$ ).

The other issues we want to investigate, concerning the numerical results, are the following:

- Do the numerical results indicate that the expected value of the solution $E\left(y_{N}(t)\right)$ of $(17)$ converges to a certain function, as $N$ tends to $\infty$ ? (Here $y_{N}$ denotes the numerical solution obtained with $h=1 / N$ ). With the aim of answering this question, we have carried out a number of simulations, using different values of $\gamma$ and $\alpha$. We repeated each simulation with $N=8, N=16$, and $N=32$. In each case, the expected value of the solution $E\left(y_{N}\right)$ was computed based on $n s=50000$ trajectories. 
Since the expected values of the solution are unknown, in order to estimate the convergence order we use the ratio:

$$
r=\frac{\left|E\left(y_{16}\right)-E\left(y_{8}\right)\right|}{\left|E\left(y_{32}\right)-E\left(y_{16}\right)\right|}
$$

where the norm used is the maximum norm. According to the theory for the Euler-Maruyama method for ordinary stochastic differential equations [12] the order of weak convergence of this method is 1 . If the same convergence order was observed for the equation considered here, we would obtain $r \approx 2$ (as is obtained for the deterministic equation, when the method (10) is applied). If $\alpha$ and $\gamma$ lie in the intervals $[0.2,0.3]$ and $[0,0.1]$, respectively, and the number of trajectories $n s$ is sufficiently high, then we can show that $r$ is close to 2, indicating first order weak convergence of the method. In Table 1, the values of the ratio $r$ are displayed for different values of $\alpha$ and $\gamma$.

Note that to obtain good estimates of the weak convergence order the sampling error of the results should be negligible in comparison with the discretisation one. In our computations the smallest discretisation step is $h=\tau / 64 \approx 0.01$. Since the sampling error is known to decrease as $1 / \sqrt{n s}$, we should choose a number of simulations $n s$ so that $n s>>1 / h^{2}$. This is why we have used, for Tables 1 and $3, n s=50000$. However, even with this value of $n s$, in some cases the estimates of the convergence order are not close to 2 . More accurate estimates could probably be obtained by using a greater value of $n s$.

- How do the expected value and the minimal and maximal trajectories of the solutions of (17) depend on $\gamma$ and $\alpha$ ?

Before answering this question, we look at the solutions of the deterministic equation (19). When $\gamma$ is negative, the solutions of the deterministic equation (19) increase from $\alpha$ (threshold potential) to a value close to 1 ; when $\gamma$ is positive, they decrease to a value, close to 0 . Therefore, the slopes of the graphs, close to $-3 \tau$, are positive, in the case of negative $\gamma$, and negative, in the case of positive $\gamma$.

When we increase $\alpha$, for a fixed positive value of $\gamma$, the slopes become steeper (see Fig. 2). But if $\gamma$ is negative, the slopes become less steep, as $\alpha$ increases (see Fig. 3).

Now, concerning the solutions of the stochastic equation, we observe that the graphs of $y_{\text {min }}$ are decreasing and their slopes become steeper, as $\alpha$ increases (see Fig.4). For this figure and the two following ones, we have carried out 100 simulations with $N=20$. In the case of $y_{\max }$, the trajectories are increasing and their gradients increase as $\alpha$ increases, though in this case the variation is not so significant (Fig. 5). Concerning the expected value of the solution $E(y(t))$ (see Fig.6), it increases with $t$ and for most values of $\alpha$ tends to 0.5 (the average between 0 and 1 ). This is the reason for the second boundary condition in (20). The slopes increase with $\alpha$.

The behaviour of the solutions of the problem may also be analysed by comparing the estimated expected values of the local growth rate at a certain point, inside the layer where the solution changes fast. The approximate value of the local growth rate with $t=t_{i}$, is obtained from the formula

$$
\frac{E\left(y_{i+1}\right)-E\left(y_{i}\right)}{h}
$$

In Table 2 the approximations of the growth rate at $t_{i}=-23 / 16$ are displayed, for different values of $\alpha$ and $\gamma$. These approximations were obtained using a numerical solution with $N=64$. These results are in agreement with the above discussion on the behaviour of the expected values of the solution.

- How do the expected values of the solution of (17) compare with the solution of the deterministic equation (19)? 


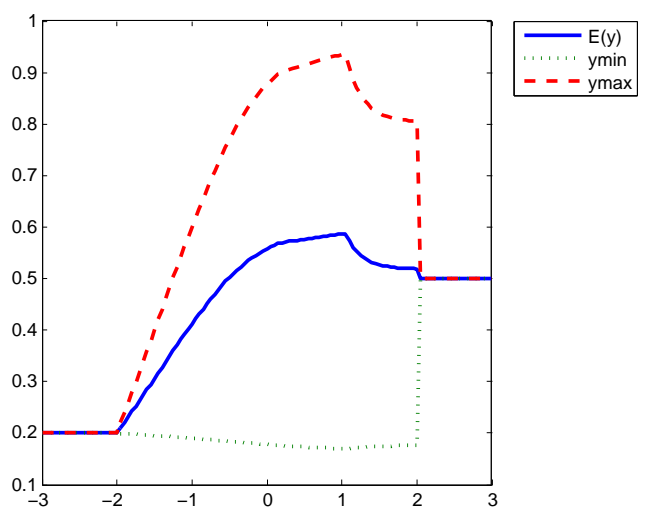

Figure 1: Solutions of the stochastic equation (15), with constant boundary conditions, $\alpha=0.2$. The trajectories were obtained with $N=20$ and $n s=100$. This figure shows that even with a small level of noise $(\gamma=0.001)$ the constant solution of the non-perturbed deterministic equation $(y(t) \equiv \alpha)$ can be transformed into a completely different function.

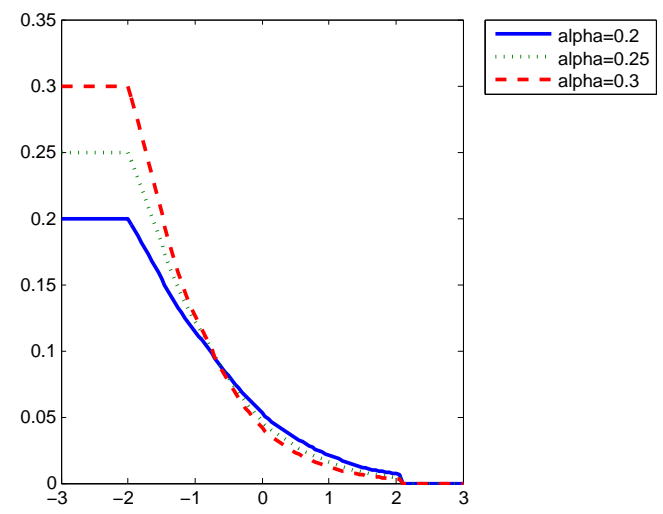

Figure 2: Solutions of the perturbed deterministic equation (19) with $\gamma=0.001$ and different values of $\alpha$. These solutions have a behaviour similar to $y_{\min }$ in Fig.1.
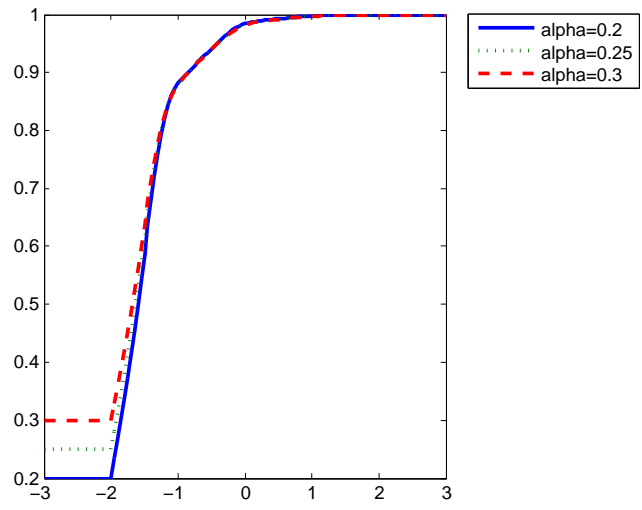

Figure 3: Solutions of the deterministic equation (19) with $\gamma=-0.001$ and different values of $\alpha$. These solutions have a behaviour similar to $y_{\max }$ in Fig.1. 

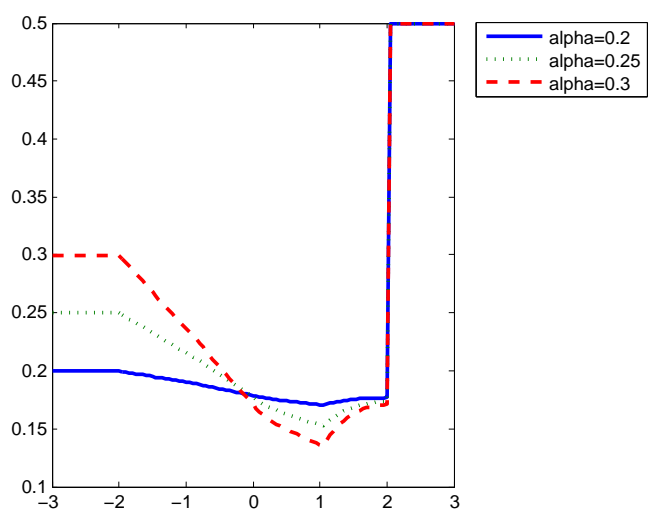

Figure 4: Graphs of $y_{\min }$, for equation (15), in the case $\gamma=0.001$ with different values of $\alpha$. The functions are decreasing on $[-2,2]$ and the slopes of the graphs increase with $\alpha$.

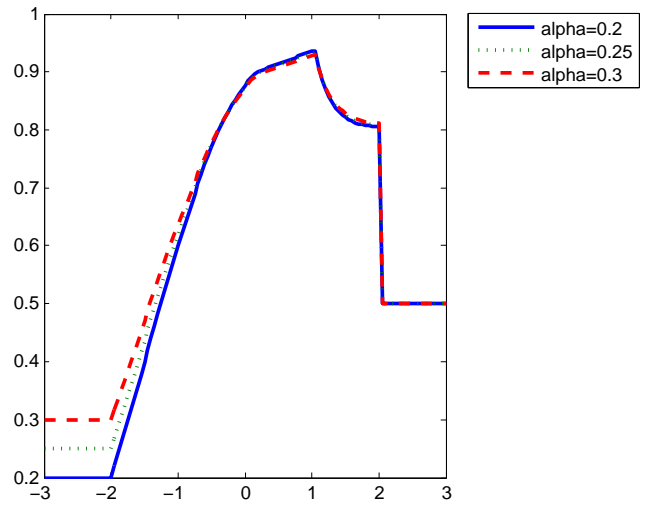

Figure 5: Graphs of $y_{\max }$, for equation (15), in the case $\gamma=0.001$, with different values of $\alpha$. The functions are increasing on $[-2,2]$ and the slopes of the graphs decrease with $\alpha$.

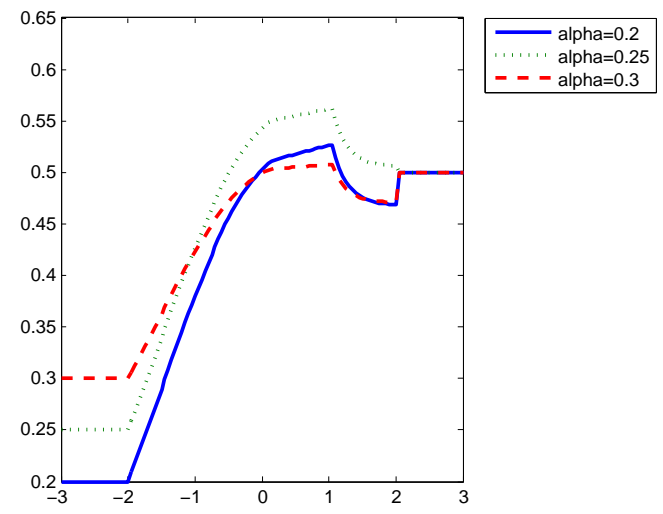

Figure 6: Graphs of $E(y(t))$, for equation (15), in the case $\gamma=0.001$, with constant boundary conditions and different values of $\alpha$. 
In the previous paragraphs, we have partially answered this question. For a fixed value of $\gamma$, the expected values of the solution behave approximately like the average of the deterministic solution corresponding to this value of $\gamma$ and the solution corresponding to $-\gamma$ (compare Fig.6 with Fig.2 and Fig.3).

In some of the graphs, we observe that the behaviour of the estimated expected solution is nonregular in a certain sense: the solution may be non-monotone in the whole interval and it may have a jump in the local growth rate at some points (the so-called break points). This results from the fact that we are considering $\tau=1$ in all the simulations. In order to get smooth solutions of equation (15), the correct value of $\tau$ must be found for each set of values $B, \alpha, \gamma$. Here we did not try to compute this value, which results in the solution irregularities. In section 4.2, we will carry out some simulations where the correct values of $\tau$ are taken from other sources and the solutions we obtain don't have this kind of irregularity.

\subsection{Solving the stochastic equation using asymptotic boundary conditions}

In Sec. 2 we described how to build forward and backward solutions of equation (3), using the boundary conditions (6) and (7), which follow from the asymptotic analysis of the solutions. In this subsection, we will report the results of numerical experiments, where solutions were obtained numerically for the stochastic equation (15), using the Euler-Maruyama method in the form (17) or (18).

As described in Sec. 2, the correct values of $\tau, \lambda_{-}, \lambda_{+}$must be computed for each set of values $\alpha, A, B$, in order to construct a smooth increasing solution of (3). Such values were obtained in [10] and we shall use them to build the solutions of the stochastic equation.

Our main goal is to compare the numerical solutions of the stochastic equation (15) with those of the corresponding deterministic equation (3). Note that these last solutions can be obtained using the methods (9) or (10), or equivalently, applying the methods (17) or (18) with $\gamma=0$. In this way, we may analyse how far a stochastic perturbation can affect the solutions of the deterministic equation, whose properties have been studied before.

The numerical experiments have shown that, in order to preserve the convergence of the iterative procedure and obtain sufficiently accurate results, one should apply formulae (17) and (18) to intervals that are not very large. Therefore, we have chosen to apply each formula, (17) or (18), on the intervals $[-2 \tau, 0]$ and $[0,2 \tau]$ separately; in the first case, boundary conditions are given on $[-3 \tau,-2 \tau]$ and $[0, \tau]$; in the second case, on $[2 \tau, 3 \tau]$ and $[-\tau, 0]$. The boundary conditions, as well as the initial approximation for the iterative process, are taken from the results in $[10]$.

- As in the previous subsection, we begin by addressing the problem of convergence. Therefore, we solve each problem with at least three different stepsizes and we compute the ratio (23).

The results of these computations, for different values of $\alpha$ and $\gamma$, are displayed in Table 3. These results were obtained solving the stochastic equation on the positive semiaxis. Similar results can be obtained using the same equation on the negative semiaxis.

- The second question we analyse is how the expected values of the solution $E(y(t))$, the minimal trajectory $y_{\min }(t)$ and maximal one $y_{\max }(t)$ depend on $\alpha, A, B$, and $\gamma$. The computations for the graphs of Fig. 7-10 were carried out with $N=32$ and $n s=100$. We have considered $A=1$ and $B=15$ (except where different values of $B$ are specified).

In Fig. 7 the expected value of the solution on the interval $[-\tau, 3 \tau]$ is displayed, as well as the minimal and maximal trajectories, in the case $\alpha=0.1, B=15, \gamma=0.1$. In this case, we have $\tau=0.50558$. For the interval $[-3 \tau, \tau]$, the corresponding graphs are depicted in Fig. 8. We can see that the expected value of the solution in both cases has a regular behaviour, similar to the one of the solution in the 
deterministic case. From the graphs of Fig. 7 and Fig. 8, we can see that the solutions are much more strongly affected by the noise on the interval $[-\tau, 3 \tau]$ than on $[-3 \tau, \tau]$.

The graphs of $y_{\min }$ and $y_{\max }$, on the interval $[-\tau, 3 \tau]$, for different values of $\gamma$, can be found in Fig. 9 and Fig.10, respectively. We observe the values of $y_{\min }$ decrease as $\gamma$ increases, while the values of $y_{\max }$ increase. As $\gamma$ increases we observe that $y_{\max }$ can get values much higher than 1 (the solution maximum in the deterministic case), while $y_{\min }$ loses monotonicity.

The tables 4-6 indicate some other properties of the solutions. In Table 4 we compare the expected value of the solution at $t=0(E(y(0)))$ with $y_{\min }(0)$ and $y_{\max }(0)$; the values are given for the case $\alpha=0.1$, with $\gamma=0.1,0.2,0.3$. The results confirm that the difference between $y_{\min }(0)$ and $y_{\max }(0)$ increases quickly with $\gamma$; the expected value of $y(0)$ also increases with $\gamma$, but only slowly. Similar results are displayed in Table 5 for the case of $\alpha=0.15$. Comparing the two tables, we conclude that the dispersion between solutions of the stochastic equation is smaller for $\alpha=0.15$ than for $\alpha=0.1$. The results of both tables were obtained by approximating the solution on $[-2 \tau, 0]$. If we approximate the solution on $[0,2 \tau]$, we can obtain approximations of $E(y(2 \tau)), y_{\min }(2 \tau)$ and $y_{\max }(2 \tau)$. Such results are presented in Tables 6 and and 7, for $\alpha=0.1$ and $\alpha=0.15$, respectively. Comparing the last two tables with the first two ones, we confirm that the solutions are much more affected by noise on the interval $[0,2 \tau]$ than on $[-2 \tau, 0]$. For all values of $\alpha$ and $\gamma$, the difference $y_{\max }(2 \tau)-y_{\min }(2 \tau)$ is always much greater than $y_{\max }(0)-y_{\min }(0)$. Moreover, as in the case of Table 4 and Table 5 , this difference increases with $\gamma$ and decreases with $\alpha$.

Finally, it is interesting to analyse how the local growth rate of $E(y(t))$ at a certain point (se formula (24)) depends on $\alpha$ and $\gamma$. In Table 8, the approximate values for $t=2 \tau$ are displayed, for different values of $\alpha$ and $\gamma$. From this table, we conclude that the slope of the curves is decreasing with respect both to $\alpha$ and $\gamma$.

\section{Conclusions and Future Work}

The main purpose of this paper is to introduce the stochastic equation (1) and compare the dynamical behaviour of its solutions with the solutions of the corresponding deterministic equation (2), studied before.

Since there have been no previous attempts at solving stochastic mixed-type functional differential equations. (as far as we are aware), we have created a new computational method to approximate the solutions of (1). This method is described in Sec. 2 and Sec. 3. As initial approximations, we have used approximate solutions of the deterministic equations, obtained by known methods, and then adapted the Euler-Maruyama method to deal with the stochastic equation. This results in an iterative process, which converges in most cases of practical interest.

The convergence of the discretisation method has been tested experimentally and the obtained estimates of the weak convergence rate (close to 1) are in agreement with the known theory about the Euler-Maruyama method.

Concerning the dynamical behaviour of the solutions of equation (1), our numerical experiments lead to the following conclusions.

- When equation (1) is solved with constant boundary conditions $(y(t) \equiv \alpha$, which is an unstable equilibrium point for the deterministic equation(2)), we observe that the solutions are very sensitive to the stochastic noise, whose level is governed by $\gamma$. Independent of the value of $\alpha$, even with $\gamma=0.001$, the trajectories of (1) may take almost any value on $[0,1]$, with the expected solution close to 0.5 , as $t \rightarrow \infty$.

This is not surprising, when we compare with the solutions of a modified version of the deterministic equation (2). When a small perturbation of the form $\gamma y(t)(1-y(t))$ is added to its right-hand side, 


\begin{tabular}{|c|c|c|c|}
\hline$\alpha$ & 0.2 & 0.25 & 0.3 \\
\hline$\gamma=0.001$ & 1.254 & 2.2920 & 2.3099 \\
$\gamma=0.01$ & 2.2969 & 2.4412 & 2.5088 \\
$\gamma=0.1$ & 2.4391 & 1.3179 & 1.9850 \\
\hline
\end{tabular}

Table 1: values of the coefficient (23), indicating the weak convergence order in the case of constant boundary conditions.

\begin{tabular}{|c|c|c|c|}
\hline$\alpha$ & 0.2 & 0.25 & 0.3 \\
\hline$\gamma=0.001$ & 0.4032 & 0.3456 & 0.2752 \\
$\gamma=0.01$ & 0.4038 & 0.3392 & 0.2688 \\
$\gamma=0.1$ & 0.4096 & 0.3392 & 0.2688 \\
\hline
\end{tabular}

Table 2: Estimated expected values of the local growth rate at $t=-23 / 16$, in the case of constant boundary conditions.

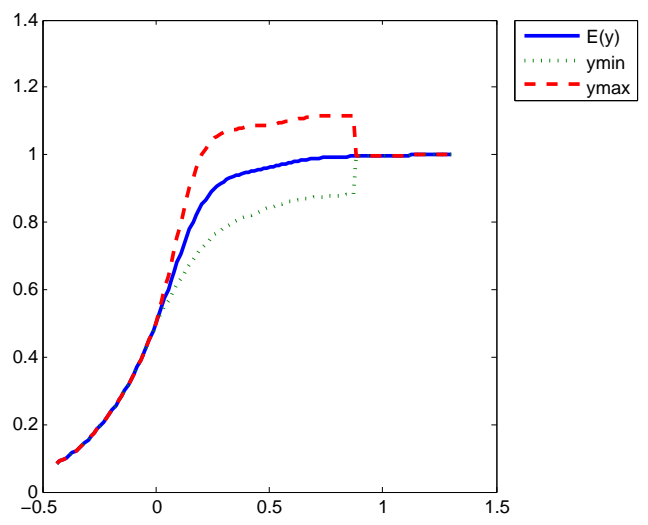

Figure 7: Graphs of $E(y(t)), y_{\min }$ and $y_{\max }$ for equation (15) on $[-\tau, 3 \tau]$, in the case of boundary conditions $(5)$, with $\gamma=0.1$, $\alpha=0.1$. The three trajectories have a similar behaviour to the soution of the deterministic equation, and the effect of noise is rather strong.

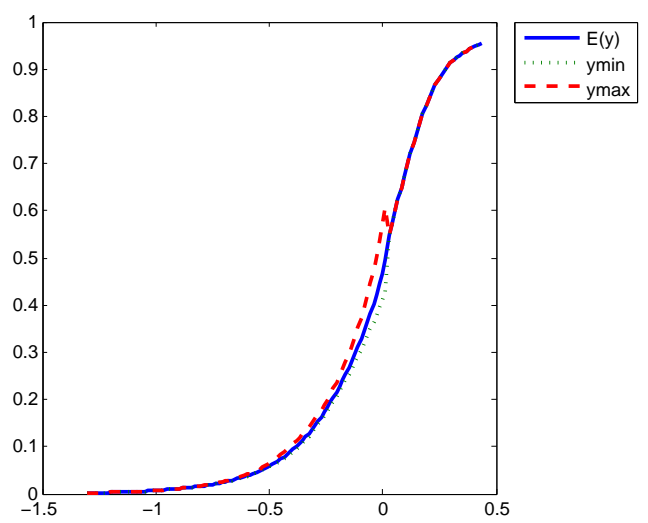

Figure 8: Graphs of $E(y(t)), y_{\min }$ and $y_{\max }$ for equation (15) on $[-3 \tau, \tau]$, in the case of boundary conditions (5), with $\gamma=0.1$, $\alpha=0.1$. The three trajectories have a similar behaviour to the solution of the deterministic equation, but the effect of noise is not so strong as in the case of the interval $[-\tau, 3 \tau]$. 


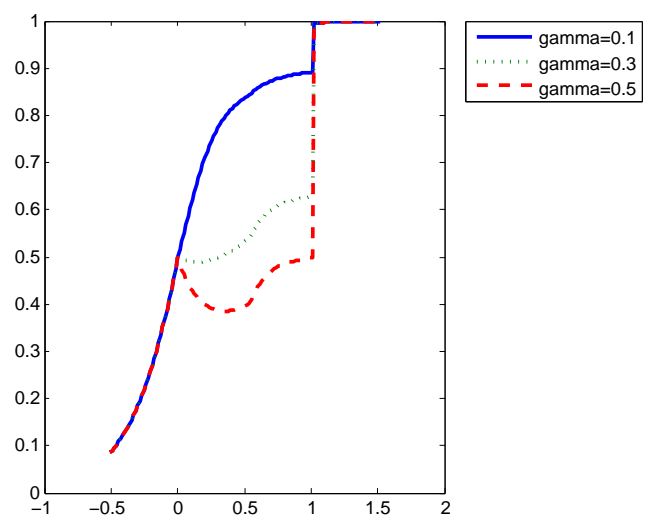

Figure 9: Graphs of $y_{\min }$ in the case $\alpha=0.1$, on $[-\tau, 3 \tau]$, with different values of $\gamma$. As $\gamma$ increases, the trajectories lose monotonicity.

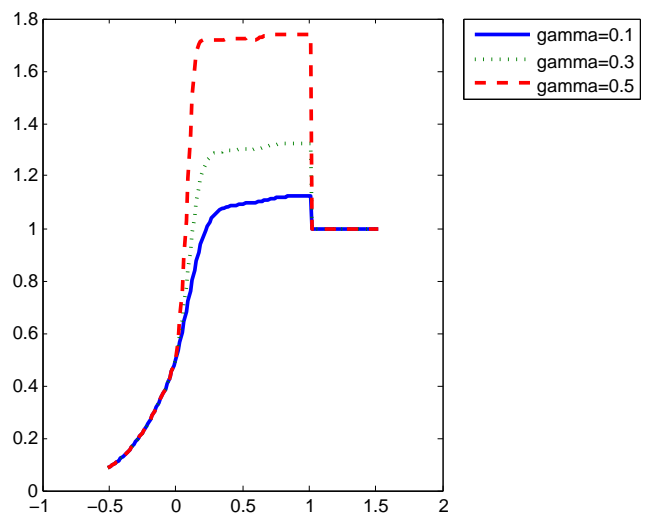

Figure 10: Graphs of $y_{\max }$ in the case $\alpha=0.1$, on $[-\tau, 3 \tau]$, with different values of $\gamma$. As $\gamma$ increases, $y_{\max }$ can take values much higher than 1 (the solution maximum in the deterministic case).

\begin{tabular}{|c|c|c|c|}
\hline$\alpha$ & 0.01 & 0.05 & 0.15 \\
\hline$\gamma=0.1$ & 2.2581 & 2.4960 & 2.3602 \\
$\gamma=0.2$ & 4.4782 & 2.7778 & 2.4371 \\
$\gamma=0.3$ & 2.1553 & 1.1684 & 1.3371 \\
\hline
\end{tabular}

Table 3: Values of the coefficient (23), indicating the weak convergence order, in the case of asymptotic boundary conditions.

\begin{tabular}{|c|c|c|c|}
\hline$\gamma$ & $E(y(0))$ & $y_{\min }(0)$ & $y_{\max }(0)$ \\
\hline 0.1 & 0.3347 & 0.3189 & 0.3645 \\
0.2 & 0.3370 & 0.2986 & 0.3841 \\
0.3 & 0.3373 & 0.2797 & 0.4717 \\
\hline
\end{tabular}

Table 4: Expected solution, minimal and maximal value at $t=0$, for equation (15) with $\alpha=0.1$. 


\begin{tabular}{|c|c|c|c|}
\hline$\gamma$ & $E(y(0))$ & $y_{\min }(0)$ & $y_{\max }(0)$ \\
\hline 0.1 & 0.3430 & 0.3269 & 0.3626 \\
0.2 & 0.3435 & 0.3134 & 0.3773 \\
0.3 & 0.3437 & 0.3049 & 0.4160 \\
\hline
\end{tabular}

Table 5: Expected solution, minimal and maximal value at $t=0$, for equation (15) with $\alpha=0.15$.

\begin{tabular}{|c|c|c|c|}
\hline$\gamma$ & $E(y(2 \tau))$ & $y_{\min }(2 \tau)$ & $y_{\max }(2 \tau)$ \\
\hline 0.1 & 0.9434 & 0.6154 & 1.4836 \\
0.2 & 0.9671 & 0.4705 & 2.0555 \\
0.3 & 0.9863 & 0.4004 & 2.3284 \\
\hline
\end{tabular}

Table 6: Expected solution, minimal and maximal value at $t=2 \tau$, for equation (15) with $\alpha=0.1$.

\begin{tabular}{|c|c|c|c|}
\hline$\gamma$ & $E(y(2 \tau))$ & $y_{\min }(2 \tau)$ & $y_{\max }(2 \tau)$ \\
\hline 0.1 & 0.9415 & 0.6135 & 1.3944 \\
0.2 & 0.9694 & 0.5019 & 1.9563 \\
0.3 & 1.0137 & 0.3744 & 2.1840 \\
\hline
\end{tabular}

Table 7: Expected solution, minimal and maximal value at $t=2 \tau$, for equation (15) with $\alpha=0.15$.

\begin{tabular}{|c|c|c|c|}
\hline$\gamma$ & $\alpha=0.05$ & $\alpha=0.1$ & $\alpha=0.15$ \\
\hline$\gamma=0.1$ & 0.1587 & 0.1509 & 0.1422 \\
$\gamma=0.2$ & 0.1410 & 0.1370 & 0.1292 \\
$\gamma=0.3$ & 0.1239 & 0.1183 & 0.1146 \\
\hline
\end{tabular}

Table 8: The local growth rate of $E(y(t))$ for equation (15), at $t=2 \tau$. 
the unstable solution $y(t) \equiv \alpha$ is transformed into a solution which tends to 0 (if $\gamma$ is positive) or to 1 (if $\gamma$ is negative). We remark that $y(t) \equiv 0$ and $y(t) \equiv 1$ are stable solutions of the equation $(2)$.

- Concerning the more realistic case of equation (1), when the boundary conditions have the correct asymptotic behaviour, we have analysed the properties of the solutions separately on the intervals $[-2 \tau, 0]$ and $[0,2 \tau]$. An important conclusion is that the solutions are much more sensitive to stochastic noise in the second case than in the first one. This could be expected from the behaviour of the monotone solutions of the deterministic equation (2), whose first derivative attains its maximal value close to $t=0$. Therefore, when the stochastic noise is introduced on the interval $[0,2 \tau]$, we start to compute the approximate solution from $t=0$, when the slope is high, and this increases the probability of the noise being amplified at $t>0$.

The other important conclusion is that the expected solutions of the stochastic equation $E(y(t))$ are smooth and increasing, and even when $\gamma=0.5$ they do not differ strongly from the solutions of the corresponding deterministic equation (when $\gamma=0$ ).

We realise that our insight into the dynamical behaviour of the solutions of equation (1) is still very limited and there are many open questions to be solved.

In the future we intend to carry out a detailed statistical analysis of the numerical results for equation (1), in particular, to investigate in detail the dependence of the expected solution and the mean deviation on $\gamma$ and on the other equation parameters.

\section{Acknowledgements}

The work presented in this paper was supported by a University of Chester International Research Excellence Award under the Santander Universities scheme.

\section{References}

[1] K. A. Abell, C.E. Elmer, A.R. Humphries, E.S. Van Vleck, Computation of mixed type functional differential boundary value problems, SIAM J. Appl. Dyn. Syst. 4 (3) (2005) 755-781.

[2] C.T.H. Baker, E. Buckwar, Numerical analysis of explicit one-step methods for stochastic delay differential equations, LMS J. Comput. Math., 3 (2000) 315-335.

[3] C.T.H. Baker, J.M. Ford, and N.J. Ford, Bifurcations in approximate solutions of stochastic delay differential equations, Int. J. Bifurcation and Chaos, 14 (2004) 2999-3021.

[4] J. Bao and C. Yuan, Convergence rate of EM scheme for SDDEs, Proc. Amer. Math. Soc. 141 (2013) 3231-3243.

[5] E. Buckwar, R. Winkler, Multi-step Maruyama methods for stochastic delay differential equations, Stochastic Analysis and Applications 25 (2007) 933-959.

[6] H. Chi, J. Bell and B. Hassard, Numerical solution of a nonlinear advance-delay-differential equation from nerve conduction theory, J. Math. Biol. 24 (1986) 583-601.

[7] R. FitzHugh, Impulses and physiological states in theoretical models of nerve membrane, Biophysical J.1(1961) $445-466$.

[8] R. FitzHugh, Computation of impulse initiation and saltatory condition in a myelinated nerve fiber, Biophysical J. 2 (1962) 11-21.

[9] N.J. Ford, P.M. Lumb, Mixed-type functional differental equations: a numerical approach, J. Comput. Appl. Math. 229 (2009) 471-479.

[10] N.J. Ford, P.M. Lima, and P.M. Lumb, Computational methods for a mathematical model of propagation of nerve impulses in myelinated axons, Applied Numerical Mathematics 85(2014) 38-53.

[11] N.J. Ford, P.M. Lumb, P. Lima, M.F. Teodoro, The numerical solution of forward-backward equations: decomposition and related issues, J. Comput. Appl. Math. 234 (2010) 2745-2756.

[12] D. J. Higham, An algorithmic introduction to numerical simulation of stochastic differential equations, SIAM Review 3 (2001) 525-546.

[13] A. Hodgkin and A. Huxley, A quantitative description of nerve current and its application to conduction and excitation in nerve, J. Physiology 117 (1952) 500-544.

[14] H.Hupkes and S.Verduyn Lunel, Center manifold theory for functional differential equations of mixed type, J. Dyn. Diff. Eq. 19 (2007) 497-560. 
[15] P.Kloeden and T. Shardlow, The Milstein scheme for stochastic delay differential equations without using anticipative calculus, Stochastic Analysis and Applications 30 (2012) 181-202.

[16] Okyu Kwon, Hie-Tae Moon, Coherence resonance in small-world networks of excitable cells, Physics Letters A 298 (2002) 319-324.

[17] P.M.Lima, M.F.Teodoro, N.J.Ford and P.M.Lumb, Analytical and numerical investigation of mixed-type functional differential equations, J. Comput. Appl. Math. 234 (2010) 2826-2837.

[18] P.M. Lima, M.F. Teodoro, N.J.Ford, P. M.Lumb, Analysis and computational approximation of a forward-backward equation arising in nerve conduction, in S. Pinelas, M. Chipot, Z. Dosla (Eds.), Differential and Difference Equations with Applications, Springer Proceedings in Mathematics and Statistics, Volume 47, 2013, 475-483.

[19] John F. Lindner et al., Array Enhanced Stochastic Resonance and Spatiotemporal Synchronization, Phys. Rev. Lett. 75 (1995), 3-6.

[20] M. Liu, C. Wanrong, and F. Zhacheng, Convergence and stability of the semiimplicit Euler method for a linear stochastic differential delay equation, J. Comput. Appl. Math. 170 (2004) 255-268.

[21] J. Mallet-Paret, The Fredholm alternative for functional differential equations of mixed type, J. Dyn. Diff. Eq. 11 (1999) $1-47$.

[22] J. Mallet-Paret and S.M. Verduyn Lunel, Mixed-type functional differential equations, holomorphic factorization and applications, in proceedings of Equadiff 2003, International Conference on Differential Equations, HASSELT 2003, World Scientific, Singapore (2005), 73-89.

[23] X. Mao, Numerical solutions of stochastic functional differential equations, LMS J. Comput. Math 6 (2003) $141-161$.

[24] X. Mao and S. Sabanis, Numerical solutions of stochastic differential delay equations under local Lipschitz condition, J. Comput. Appl. Math., 151 (2003), 215-227.

[25] J. Nagumo, S.Arimoto and S. Yoshizawa, An active pulse transmission line simulating nerve axon, Proceedings of the IRE, 50 (1962), 2061-2070.

[26] S. J. Norton(2008), Noise induced changes to dynamic behaviour of stochastic delay differential equations. (Unpublished doctoral dissertation available at http://chesterrep.openrepository.com), University of Liverpool, United Kingdom.

[27] M. Perc, Spatial coherence resonance in neuronal media with discrete local dynamics, Chaos, Solitons and Fractals 31 (2007) 64-69.

[28] M. Perc, Effects of small-world connectivity on noise-induced temporal and spatial order in neural media, Chaos, Solitons and Fractals 31 (2007) 280-291.

[29] M. Perc, Spatial decoherence induced by small-world connectivity in excitable media, New Journal of Physics 7 (2005) $1-10$.

[30] A. Rustichini, Functional differential equations of mixed type: the linear autonomous case, J. Dyn. Diff. Eq. 1 (1989) $121-143$.

[31] A. Rustichini, Hopf bifurcation for functional differential equations of mixed type, J. Dyn. Diff. Eq. 1 (1989) $145-177$.

[32] M.F. Teodoro, N.J. Ford, P.M. Lima, P. M. Lumb, New approach to the numerical solution of forward-backward equations, Front. Math. China V.4, N.1 (2009) 155-168.

[33] C. Zhou, J. Kurths, and B. Hu, Array-enhanced coherence resonance: nontrivial effects of heterogeneity and spatial independence of noise, Phys. Rev. Lett. 87 (9) (2001) 98101,1-4. 